\title{
Targeted sequencing panels in Italian ALS patients support different etiologies in the ALS/FTD continuum
}

\author{
Anna Bartoletti-Stella ${ }^{1}$. Veria Vacchiano ${ }^{1}$ - Silvia De Pasqua ${ }^{2} \cdot$ Giacomo Mengozzi $^{1}$ - Dario De Biase ${ }^{3}$. \\ Ilaria Bartolomei ${ }^{1}$ Patrizia Avoni ${ }^{1,2}$. Giovanni Rizzo ${ }^{1,2}$. Piero Parchi ${ }^{1,4}$ - Vincenzo Donadio ${ }^{1}$ - Adriano Chiò ${ }^{5,6,7}$. \\ Annalisa Pession ${ }^{3} \cdot$ Federico Oppi $^{1} \cdot$ Fabrizio Salvi $^{1} \cdot$ Rocco Liguori $^{1,2} \cdot$ Sabina Capellari ${ }^{1,2}$ on behalf of BoReALS
}

Received: 12 December 2020 / Accepted: 12 March 2021 / Published online: 26 March 2021

(c) The Author(s) 2021

\begin{abstract}
Background $5-10 \%$ of amyotrophic lateral sclerosis (ALS) patients presented a positive family history (fALS). More than 30 genes have been identified in association with ALS/frontotemporal dementia (FTD) spectrum, with four major genes accounting for $60-70 \%$ of fALS. In this paper, we aimed to assess the contribution to the pathogenesis of major and rare ALS/FTD genes in ALS patients.

Methods We analyzed ALS and ALS/FTD associated genes by direct sequencing or next-generation sequencing multigene panels in ALS patients.

Results Genetic abnormalities in ALS major genes included repeated expansions of hexanucleotide in C9orf72 gene (7.3\%), mutations in SOD1 (4.9\%), FUS (2.1\%), and TARDBP (2.4\%), whereas variants in rare ALS/FTD genes affected $15.5 \%$ of subjects overall, most frequently involving SQSTM1 (3.4\%), and CHMP2B (1.9\%). We found clustering of variants in ALS major genes in patients with a family history for "pure" ALS, while ALS/FTD related genes mainly occurred in patients with a family history for other neurodegenerative diseases (dementia and/or parkinsonism).

Conclusions Our data support the presence of two different genetic components underlying ALS pathogenesis, related to the presence of a family history for ALS or other neurodegenerative diseases. Thus, family history may help in optimizing the genetic screening protocol to be applied.
\end{abstract}

Keywords Amyotrophic lateral sclerosis $\cdot$ Frontotemporal degeneration $\cdot$ Next generation sequencing $\cdot$ Genetic heterogeneity $\cdot$ Mutation screening

\section{Introduction}

Anna Bartoletti-Stella and Veria Vacchiano contributed equally.

Rocco Liguori and Sabina Capellari share senior authorship.

Sabina Capellari

sabina.capellari@unibo.it

1 IRCCS Istituto Delle Scienze Neurologiche Di Bologna, Bellaria Hospital, 40139 Bologna, Italy

2 Dipartimento di Scienze Biomediche e Neuromotorie (DIBINEM), Università Di Bologna, 40123 Bologna, Italy

3 Department of Pharmacy and Biotechnology, Molecular Diagnostic Unit, University of Bologna, viale Ercolani 4/2, 40138 Bologna, Italy
Amyotrophic lateral sclerosis (ALS) is a devastating neurodegenerative disease characterized by the degeneration of upper and lower motor neurons. The risk of developing ALS peaks at 50-75 years of age and decreases subsequently. Survival is highly variable, but respiratory failure usually

4 Department of Experimental Diagnostic and Specialty Medicine (DIMES), University of Bologna, 40138 Bologna, Italy

5 Rita Levi Montalcini Department of Neuroscience, University of Turin, Turin, Italy

6 Azienda Ospedaliero Universitaria Citta Della Salute E Della Scienza Di Torino, Turin, Italy

7 Neuroscience Institute of Turin, Turin, Italy 
leads to death about 3-4 years after onset [1]. In up to $50 \%$ of cases, there are extra-motor manifestations such as behavior changes, executive dysfunctions, and language problems. In $10-15 \%$ of patients, these problems are severe enough to meet the clinical criteria for frontotemporal dementia (FTD) [2]. The combination of FTD with motor neuron disease (MND) refers to ALS/FTD [3]. The relationship between ALS and FTD has been confirmed by genetic studies, and these two conditions are now considered at the opposite extremes of a clinical-pathological continuum [4, 5].

Most cases are sporadic (sALS), but $10-20 \%$ of patients present a familial recurrence (fALS) [6], usually showing an autosomal dominant transmission [4]. Recent studies have highlighted the role of genetic risk factors even in sporadic patients, where heritability would represent about $21.0 \%$ [7], and twin studies have estimated a heritability of about $60 \%$ [8].

More than 30 genes have been linked to ALS and the ALS/FTD continuum [9], in addition to a small percentage of patients with sALS [10]. Four major genes, chromosome 9 open reading frame 72 (C9orf72), superoxide dismutase 1 (SOD1), TAR DNA-binding protein (TARDBP), and fused in sarcoma (FUS) cover up to $60 \%$ of fALS and $10-13 \%$ of sALS cases [9]. Variants in other genes are found in $<1 \%$ of patients [10,11].

An increasing number of cohort studies have investigated the relationship between genes and clinical phenotypes [12], as a model for researching the mechanisms underlying the disease onset and progression. They demonstrated that incomplete penetrance and genetic pleiotropy further complicate the scenario. Furthermore, more than one potentially pathogenic variant can be identified in a single patient, suggesting an oligogenic inheritance with a dose-dependent gene-burden effect [13, 14].

In this study, we compared the clinical and genetic data of Italian ALS patients to assess the genetic contribution to the pathogenesis of familial and sporadic ALS.

\section{Methods}

\section{Patients}

We collected clinical and genetic data from patients with definite, probable, probable laboratory-supported, and possible ALS diagnosed according to the revised El Escorial criteria [15] at the Clinica Neurologica, Bellaria Hospital (Bologna, Italy) between 2010 and 2019. Clinical data include gender, age at onset (AAO), type of onset, ALS phenotype, and family history. Regarding family history, we differentiated patients with a positive family history for ALS (fALS-ALS) from patients with a family history for other neurodegenerative diseases (fALS-ND), i.e., dementia and parkinsonism. Patients without a family history for ALS, parkinsonism or dementia were defined as sporadic (sALS).

Patients were classified into the following clinical phenotypes: classic, bulbar, predominant upper motor neuron (PUMN), and predominant lower motor neuron (PLMN) [16, 17]. Due to the relative small sample, we decided to include patients with flail arm, flail leg, and progressive muscular atrophy (PMA) variants in the PLMN group, while patients with primary lateral sclerosis were included in the PUMN category. Cognitive status was assessed through the clinical history, neurological examination, and a neuropsychological assessment including the Frontal assessment battery [18], and the Brief Mental Deterioration Battery (BMDB) [19].

For possible ALS, probable laboratory-supported ALS and probable ALS the diagnosis was confirmed during follow-up visits. The study was conducted according to the revised Declaration of Helsinki and Good Clinical Practice guidelines, and approved by the local ethics committee "Area Vasta Emilia Centro". Informed consent was given by participants.

\section{Genetic analysis}

\section{DNA extraction}

Genomic DNA (gDNA) was extracted from peripheral blood by standard procedures [13]. gDNA was quantified using the Quantus Fluorometer (Promega) with QuantiFluor doublestranded DNA system (Promega).

\section{Gene sequencing}

All 330 patients were screened for mutations in ALS major genes [20]: SOD1 (all exons), FUS (exons 6 and 15), TAR$D B P$ (exons 2, 3, and 5) genes and for pathogenic repeat expansion (RE) in the C9orf 72 gene as previously reported $[13,21]$.

Since 2015, genetic screening has been performed by next generation sequencing (NGS) multigene panels. We used one of the following custom gene panels: ampliconbased Illumina panel (TruSeq Custom Amplicon 1.5, Illumina, CA, USA) [22]; probe-based Illumina panel (Nextera Rapid Capture, Illumina); probe-based Agilent panel (Sure Select XT2, Agilent Technologies, Santa Clara, CA, USA), and Neurodegeneration Illumina panel (TruSeq Neurodegeneration, Illumina). For the purpose of this study, we analyzed only genes previously linked to ALS or ALS/FTD spectrum: $C C N F, C H C H D 10, C H M P 2 B$, DCTN1, FIG4, GRN, MAPT, OPTN, SETX, SQSTM1, TBK1, TREM2, TYROBP, UBQLN2, and VCP (Table S1). Due to the presence of patients with a family history of 
dementia, we also searched for rare pathogenic variants in genes linked to other forms of dementia: APP, PSEN1, PSEN2, ITM2B, CSF1R, and NOTCH3 genes (Table S1). Rare ALS genes (ALS2, ANG, PFN1, SPAST, TUBA4A, $U B Q L N 1$, and $V A P B)$, and ALS risk factor genes (NEFH, $N E K 1$, and $C 21$ orf 2) were analyzed by Neurodegeneration Illumina panels in 51 patients (Table S1).

Enriched libraries were sequenced using MiSeq or NextSeq sequencer (Illumina), with a paired-end approach. Data were automatically analyzed by MiSeq Reporter software (Illumina) on the instrument, or by an in-house bioinformatic pipeline (Supplementary Materials). Variants were reported using the HGVS-Sequence Variant Nomenclature.

\section{Variant classification}

Variant selection was performed with BaseSpace Variant interpreter (Illumina, CA, USA). Rare single-nucleotide variant (SNV) and small indels with a minor allele frequency (MAF) $<1 \%$ in the 1000 Genome Project database (http://browser.1000genomes.org/) or in the Genome Aggregation Database (GnomAD) [23] were selected. Variants were classified according to the American College of Medical Genetics and Genomics guidance for the interpretation of sequence variants [24]. Those reported in the ClinVar or HGMD (The Human Gene Mutation Database) databases were classified accordingly as known disease-causing variant (pathogenic) or variant of uncertain significance (VUS). Novel variants' pathogenicity was assessed with in-silico effect predictor tools (Supplementary Materials).

\section{Statistical analysis}

Statistical analysis was performed using IBM SPSS Statistics version 25 (IBM, Armonk, NY, USA). Kolmogorov-Smirnov test was used to verify the normal distribution of the data. Quantitative continuous variables were described as mean and standard deviation (SD) if the distribution was normal, or as median and range otherwise. Categorical variables were expressed as counts and percentages.

For continuous variables, the Mann-Whitney $U$ or the Kruskal-Wallis tests were used to test differences between two or more groups, respectively. The chi-squared test was adopted for categorical variables and the post hoc test with Bonferroni adjustment was used if the overall chi-squared test was significant. For statistical tests, $p<0.05$ was considered significant.

\section{Results}

\section{Clinical features}

A total of 330 Italian patients were included in the study and their clinical data are shown in Table 1. The median AAO was 63 years, ranging from 27 to 87 years (Table 1).

Overall, ALS phenotypes were distributed as follows: bulbar 5.2\%, classic 74.8\%, PLMN 10.9\%, and PUMN 9.1\%; $6.4 \%$ of all patients also showed cognitive deficits, mainly consistent with FTD. Our cohort includes $20.9 \%$ of patients with early-onset, defined as young-onset ALS (before or at the age of 50, arbitrary cut-off [25]). These patients showed a significantly higher percentage of PUMN variants than the other patients $(15.9 \%$ vs. $7.3 \%, p$ value $=0.017)$.

Table 1 Clinical features of study population

\begin{tabular}{|c|c|c|}
\hline Patients/clinical characteristics & $N$ (tot. 330) & $\%$ \\
\hline \multicolumn{3}{|l|}{ Gender } \\
\hline Male & 173 & 52.4 \\
\hline Female & 157 & 47.6 \\
\hline \multicolumn{3}{|l|}{ Age at onset (y) } \\
\hline Median (range) & $63(27-87)$ & \\
\hline \multicolumn{3}{|l|}{ Type of onset } \\
\hline Bulbar & 95 & 28.8 \\
\hline Spinal & 193 & 58.5 \\
\hline Pseudo-polyneuritic & 18 & 5.5 \\
\hline Pyramidal & 24 & 7.3 \\
\hline \multicolumn{3}{|l|}{ ALS variant } \\
\hline Classic & 244 & 74.8 \\
\hline Bulbar & 17 & 5.2 \\
\hline PLMN & 30 & 10.9 \\
\hline PUMN & 39 & 9.1 \\
\hline Deceased patients & 187 & 56.7 \\
\hline \multicolumn{3}{|l|}{ Disease duration $(\mathrm{m})$} \\
\hline Median (range) & $35(4-169)$ & \\
\hline \multicolumn{3}{|l|}{ Family history } \\
\hline fALS & 84 & 25.5 \\
\hline fALS-ALS & 30 & 9.1 \\
\hline fALS-ND & 54 & 16.4 \\
\hline sALS/unknown & 222 & 74.5 \\
\hline \multicolumn{3}{|l|}{ Other clinical features } \\
\hline Dementia & 21 & 6.4 \\
\hline
\end{tabular}

$A L S$ amyotrophic lateral sclerosis; fALS familial ALS; $f A L S$ - $A L S$ familial ALS with positive family history for ALS; $f A L S-N D$ familial ALS with positive family history for other neurodegenerative diseases; $m$ months; $N$ number; PLMN predominant lower motor neuron; $P U M N$ predominant upper motor neuron; $s A L S$ sporadic ALS 
Analysis of family history showed that $9.1 \%$ of our patients had a positive family history for ALS (fALS-ALS) (Table 1). Given the ALS/FTD continuum, we examined the percentage of patients with relatives affected by other neurodegenerative disorders, particularly dementia and/or parkinsonism (fALS-ND). These patients accounted for $16.4 \%$ of the cohort. Thus, $25.5 \%$ of our patients had a familial form of the ALS/FTD continuum [26]. Overall, fALS patients had a significantly earlier AAO (median 59 years) than sALS (median 64 years), $p$ value $=0.047$.

Stratifying the familiarity for AAO, we observed a progressive decrease in familial forms after age 60 (patients with AAO before 60 had a familial form in $34.4 \%$ of cases compared to $22.3 \%$ of patients with AAO after age $60, p$ value $=0.034 ;$ Fig. 1a). However, the trend was different between fALS-ALS and fALS-ND subgroups (Fig. 1b). Indeed, the percentage of fALS-ALS was higher in patients with young-onset ALS (18.8\% of ALS patients with $\mathrm{AAO} \leq 50$ as compared to $6.5 \%$ of patients with $\mathrm{AAO}>50$, $p$ value $=0.007$ ), while no differences were observed concerning the family history of fALS-ND.

Patients with cognitive decline showed a high percentage of positive family history for ALS and/or dementia/
A

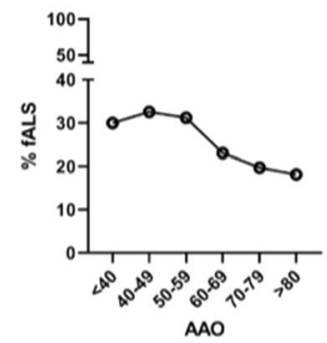

B

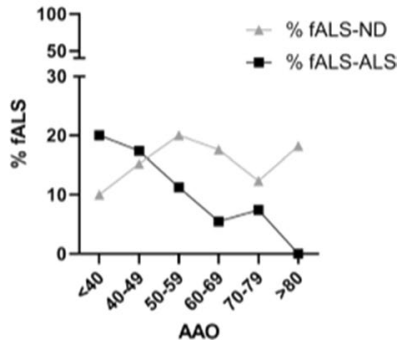

Fig. 1 Inheritance features of the study population. A, b The graph shows the percentage of ALS patients with positive family history (a, fALS); for ALS (b, fALS-ALS) and for other neurodegenerative diseases (b, fALS-ND) stratified for age at onset (AAO) parkinsonism $(55.5 \%$ vs $25.5 \%, p$ value $=0.004)$, while they did not significantly differ for AAO ( $p$ value $=0.558)$. Family history was similar among the different ALS phenotypic variants.

\section{Genetic screening}

All patients were screened for the RE in the $C 9 \operatorname{orf} 72$ gene and for mutations in SOD1, FUS, and TARDBP. 207 patients, enriched for a positive family history, were also analyzed by NGS multigene panels to verify the presence of mutations in the rare genes associated with ALS or the ALS/FTD continuum, and other dementia genes. 51 patients were also tested with a more extensive panel to test the genes reported as risk factors in ALS and other rare genes (Table S1).

\section{Genetic variants in the ALS major genes}

Fifty-five patients (16.7\%) presented a pathogenic or probably pathogenic genetic variant in one of the four major genes (Table 2). The mutation frequency was $41.7 \%$ in fALS and $8.1 \%$ in sALS. These frequencies were significantly different between fALS-ALS and fALS-ND patients $(76.7 \%$ vs. $22.2 \%, p$ value $<0.0001$ ).

The pathogenic RE in the C9orf72 gene was the most frequent mutation ( $n=24,7.3 \%$ of all cases, Table 2$)$. Among these, 18 patients (21.4\% of fALS) presented a positive family history, with no significant difference between the percentages in two subgroups of fALS, $26.7 \%$ among fALS-ALS cases, and $18.5 \%$ among fALS-ND cases ( $p$ value $=0.55)$. Six patients were sALS $(2.4 \%$ of all sALS cases). In one patient, we identified an intermediate repeat expansion (24-30 repeats) [27].

Clinical features of C9orf72 RE carriers are detailed in Table S2. We then identified 11 different variants in the SOD1 gene (Tables 2 and S3), of which five classified as definite pathogenic, five as VUS, and one (c.73-4A > G),
Table 2 Number of pathogenic mutations in ALS-major genes identified in this study

\begin{tabular}{|c|c|c|c|c|c|c|c|c|c|c|}
\hline \multirow[t]{3}{*}{ Gene } & \multicolumn{2}{|c|}{ All $(n=330)$} & \multicolumn{6}{|c|}{ fALS $(n=84)$} & \multicolumn{2}{|c|}{$\begin{array}{l}\text { sALS } \\
(n=246)\end{array}$} \\
\hline & \multirow[t]{2}{*}{$n$} & \multirow[t]{2}{*}{$\%$} & \multirow[t]{2}{*}{$n$} & \multirow[t]{2}{*}{$\%$} & \multicolumn{2}{|c|}{$f A L S-A L S(n=30)$} & \multicolumn{2}{|c|}{$f A L S-N D(n=54)$} & \multirow[t]{2}{*}{$n$} & \multirow[t]{2}{*}{$\%$} \\
\hline & & & & & $N$ & $\%$ & $n$ & $\%$ & & \\
\hline C9orf72 & 24 & 7.3 & 18 & 21.4 & 8 & 26.7 & 10 & 18.5 & 6 & 2.4 \\
\hline SOD1 & 16 & 4.9 & 8 & 9.5 & 8 & 26.7 & 0 & 0.00 & 8 & 3.3 \\
\hline FUS & 7 & 2.1 & 4 & 4.8 & 3 & 10.00 & 1 & 1.9 & 3 & 1.2 \\
\hline$T A R D B P$ & 8 & 2.4 & 5 & 6.0 & 4 & 13.3 & 1 & 1.9 & 3 & 1.2 \\
\hline Total & 55 & 16.7 & 35 & 41.7 & 23 & 76.7 & 12 & 22.2 & 20 & 8.1 \\
\hline
\end{tabular}

$A L S$ amyotrophic lateral sclerosis; $f A L S$ familial ALS; $f A L S$ - $A L S$ familial ALS with positive family history for ALS; $f A L S$ - ND familial ALS with positive family history for other neurodegenerative diseases; $n$ number; $s A L S$ sporadic ALS 
never reported, which was predicted to be likely benign (Table S4). All variants classified as VUS on HGMD or ClinVar were considered as likely causative [28-31] since for several SODI variants a reduced penetrance has been described and they can, therefore, rarely be present in controls [32]. The SOD1 variants represented the second genetic cause in our cohort ( $n=16,4.9 \%$ of all cases, Table 2$)$. Eight patients were fALS, all of them with a family history of ALS (26.7\% of fALS-ALS) and the remaining eight were sALS (3.3\% of sALS) (Table 2).

Two novel mutations were identified in the FUS gene (Table S3). Both were predicted to be damaging (Tables S3 and S4). Five variants, previously described, were identified in additional five patients (Table S3). Thus, mutations in FUS explained $2.1 \%$ of all cases, $4.8 \%$ of fALS $(10.0 \%$ fALS-ALS and $1.9 \%$ fALS-ND), and $1.2 \%$ sALS (Table 2 ).

Eight patients carried mutations in the TARDBP gene (Table 2 and S3). Variant p.Gln303His was considered likely pathogenic because it was previously described in a sALS [33]. Overall, mutations in the TARDBP gene were identified in $2.4 \%$ of our cohort, involving $6.0 \%$ of fALS $(13.3 \%$ of fALS-ALS, and $1.9 \%$ of fALS-ND, $p$ value $<0.0001$ ), and $1.2 \%$ of sALS (Table 2 ).

\section{Genetic variants identified by NGS analysis}

Among the 207 patients tested for mutations in genes rarely involved in ALS, ALS/FTD continuum, or other types of degenerative dementia (Table S1), 31 patients (14.97\% of the cohort) carried pathogenic or likely pathogenic variants (Table 2). Of the 29 different variants identified, 13 were previously reported in public databases (ClinVar or HGMD) or in the literature (Table 3). Only one variant (OPTN p.Gln314Leu) was reported as definite pathogenic; the others were classified as VUS. In addition, we identified 16 new variants considered potentially pathogenic based on the in-silico prediction of pathogenicity (Tables S4 and S5).

The genes in which we identified more variants were $\operatorname{SQSTM1}(n=7,3.4 \%$ of the cohort) and CHMP $2 B(n=4$, $1.9 \%)$. We also identified variants in: $C H C H D 10(n=2)$, CCNF $(n=1)$, DCTNI $(n=2)$, FIG4 $(n=2), \operatorname{OPTN}(n=2)$, $\operatorname{SETX}(n=3), \operatorname{TBKI}(n=2)$, and UBQLNI $(n=1)$.

Finally, we identified additional rare variants in non-FTD dementia related genes such as $A P P, C S F 1 R, I T M 2 B$, and $N O T C H 3$ (Table 3). All patients carrying variants in $A P P$, CSF1R, ITM2B (p.Ile251Val), and NOTCH3 genes showed a family history for dementia.

Four patients also carried additional pathogenic variants in one of the ALS-major genes (Table 3).

\section{Comprehensive analysis of the ALS mutation spectrum with an extended panel}

To assess genetic variants in additional ALS-related genes (PFN1, ANG, NEFH, NEK1, TUBA4A, C21orf2, and SPAST) (Table $\mathrm{S} 1)$ a subgroup of patients $(n=51)$ was screened with an extended NGS panel. Rare potentially pathogenic variants were identified in eight patients (Table 4).

In particular, we found a variant in the $N E K I$ gene in three patients, three heterozygous variants in the $A L S 2(n=3)$, and two variants in the SPAST gene, rarely linked to ALS [34]. One patient carried variants in two rare genes (NEKI and SPAST genes), two an additional pathogenic variant in SQSTM1, and C9orf72 genes (Table 4). No mutations have been identified in PFN1, ANG, NEFH, and TUBA4A genes.

\section{Genotype-phenotype correlation}

The average AAO and disease duration in patients carrying variants in the four major ALS genes, and in ALS-rare genes are shown in Table S6.

SQSTM1 was the most frequent gene carrying potentially pathogenic variants among the rare ALS genes explored (Table 3). Thus, to characterize a possible correlation with a specific phenotype, we considered this gene separately from other ALS-rare genes.

Although we did not find any significant difference between genetic groups, patients with SQSTM1 mutations presented with a mean AAO lower and more similar to that of four major ALS genes, while patients carrying rare ALS/FTD genes mutations showed an AAO comparable to that of wild-type patients. Concerning the phenotype associated with the different variants, TARDBP patients showed a bulbar phenotype more frequently than wildtype patients $(37.5 \%$ vs. $3.6 \%, p$ value $=0.0001)$, while FUS mutated patients showed a PLMN phenotype more frequently than wild-type, C9orf72 and other rare genes mutated patients $(57.1 \%$ vs. $10.8 \%, 4.2$ and $0 \%$, respectively, $p$ value $=0.0001)$.

SQSTM1 mutated patients displayed a classic phenotype without cognitive deficits at the time of diagnosis, with a family history for dementia in $28.6 \%$ of patients (Table 3 ).

Cognitive deficits were significantly more frequent in C9orf72 than in wild-type patients $(33.3 \%$ vs. $5 \%, p$ value $=0.0001$ ). No cognitive deficits were found in patients carrying variants in other genes, with the exception of three mutated patients in TARDPB (c.883G $>$ A p.Gly295Ser), TBK1 (c.225G > C p.Glu75Asp), and CHCHD 10 (c.100C $>$ T p.Pro34Ser), respectively. 


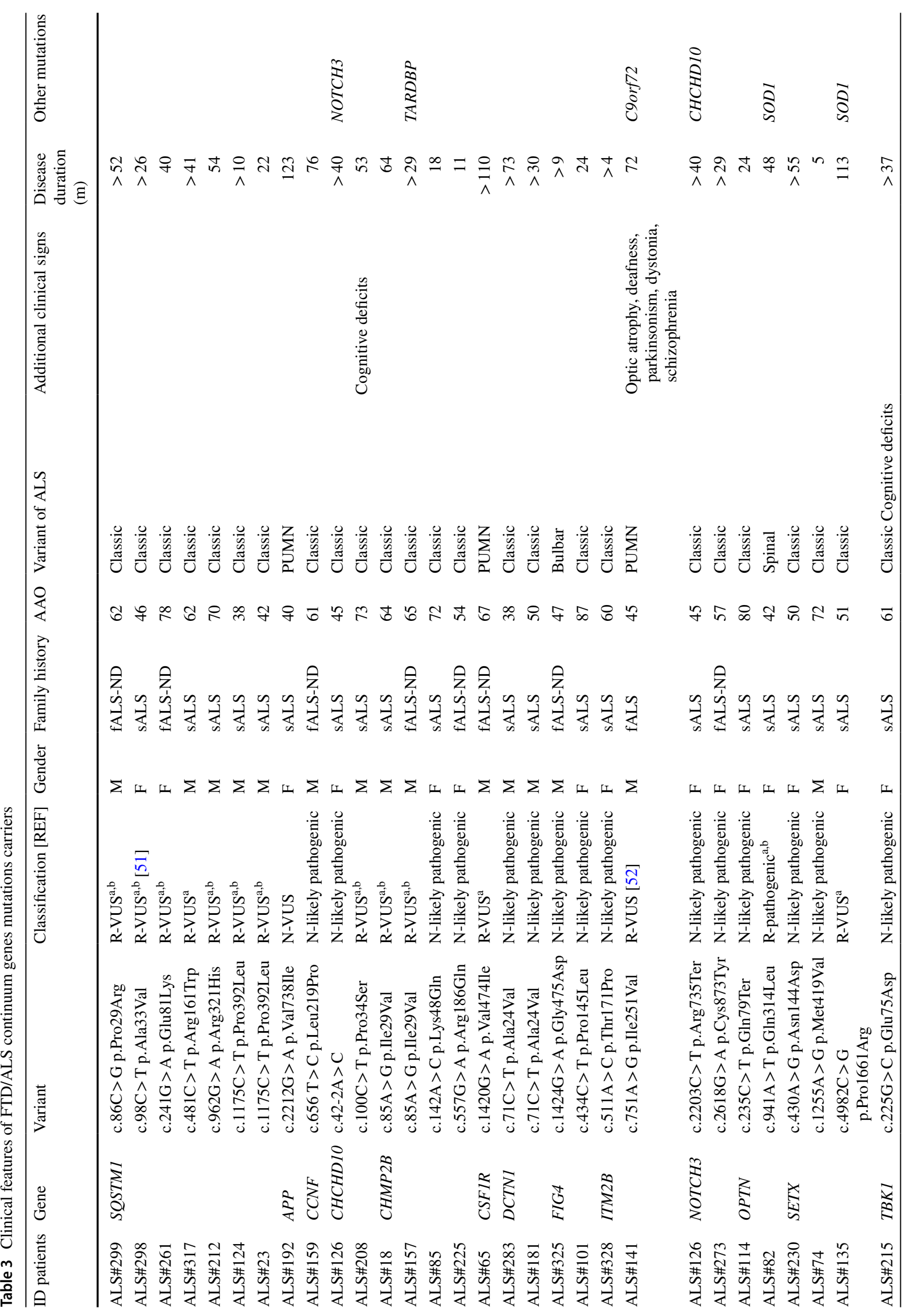




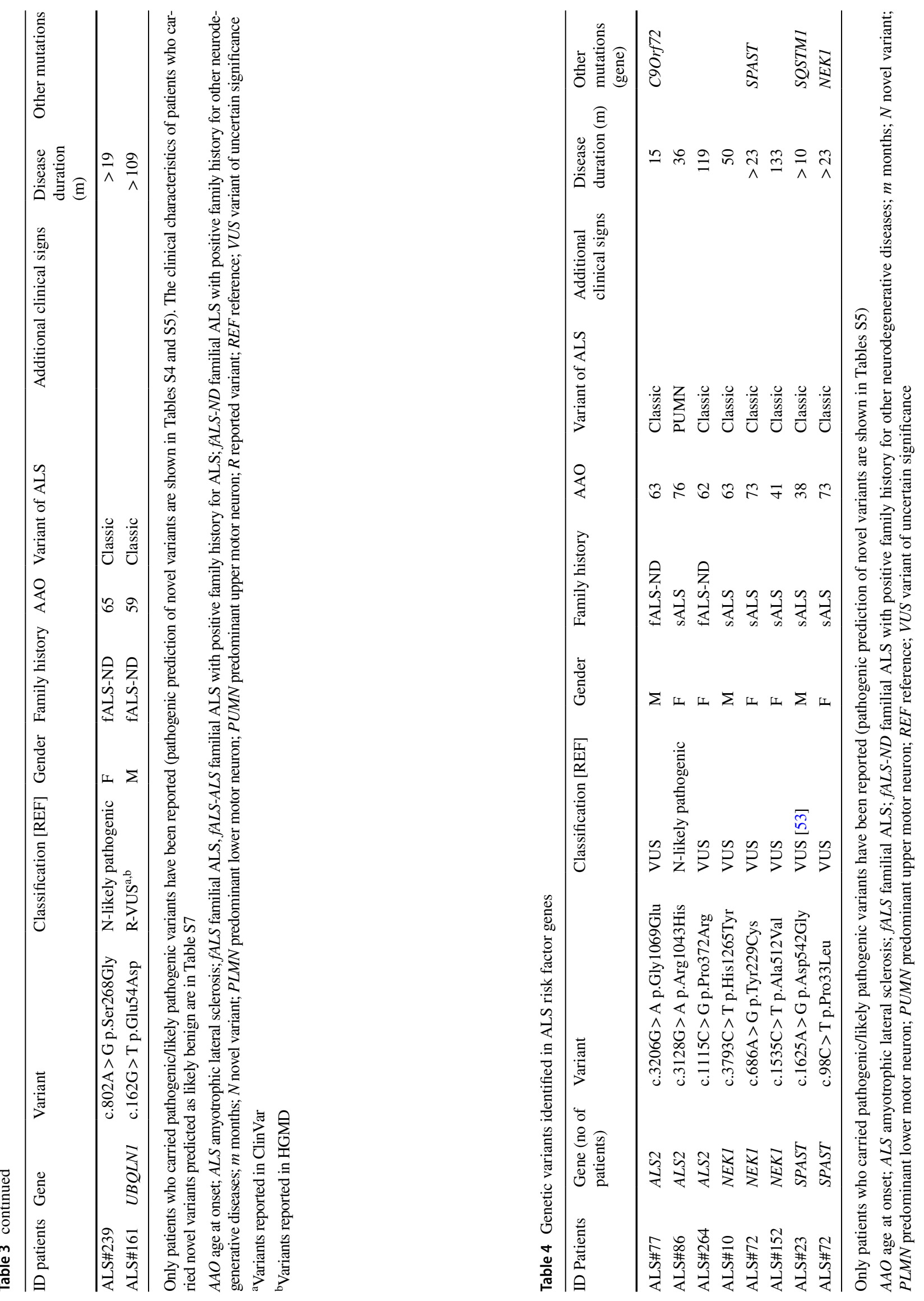


Fig. 2 Genetic variants distribution in our ALS population. Variants are classified as causative (ALS major genes and ALS/FTD genes), possibly pathogenic variants and variant of uncertain significant (VUS). The distribution of genetic variants is different between sALS and fALS patients, accounting for $17 \%$ of sALS and $55 \%$ of fALS patients. Genetic contribution was also different between fALS-ALS and fALS-ND, and between sALS, considering AAO. $A A O$ age at onset; $A L S$ amyotrophic lateral sclerosis; $f A L S$ familial ALS; $f A L S$ - $A L S$ familial ALS with positive family history for ALS; $f A L S-N D$ familial ALS with positive family history for other neurodegenerative diseases; $s A L S$ sporadic
A

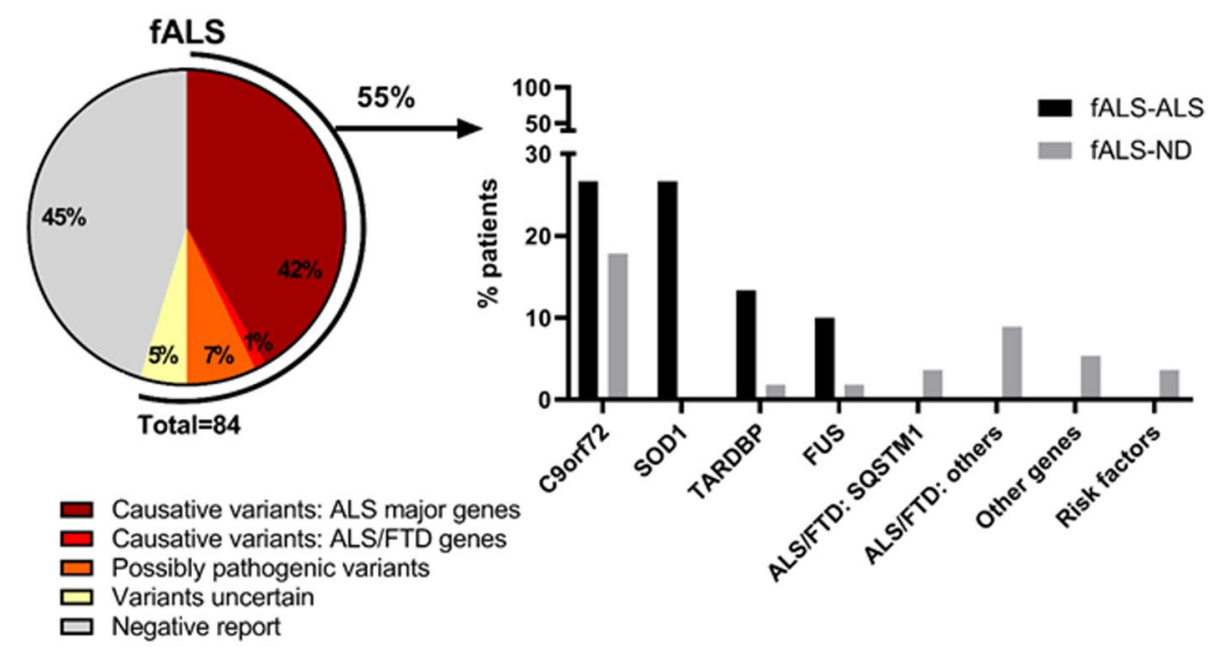

B

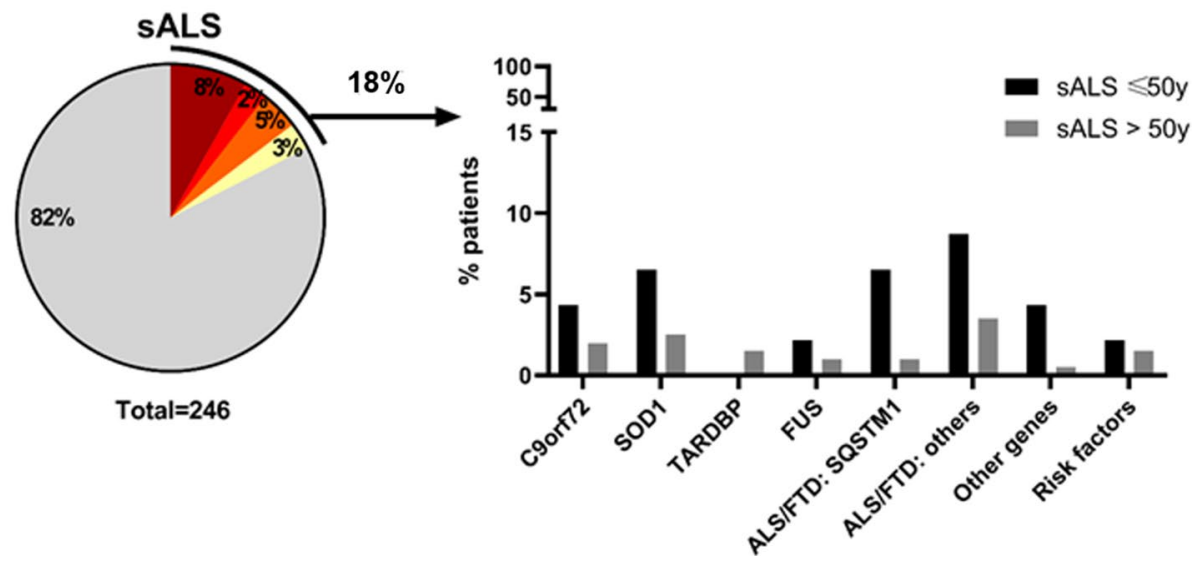

\section{Discussion}

Genetics plays an important role in ALS and FTD, recognized as two diseases that form a broad neurodegenerative continuum. At least $10 \%$ and $40 \%$ of patients diagnosed with ALS and FTD are known to carry an autosomal dominant genetic mutation [35].

Given recent advances in gene therapy [36], it has become increasingly important to predict whether and which genes are involved in single ALS patients. However, ascertaining the genetic basis in patients diagnosed with ALS and/or FTD is a challenge, given the continuous discovery of new genes rarely associated with these diseases and the detection of patients with unresolved early-onset and positive family history $[37,38]$.

In this study, we presented the family inheritance features and the genetic landscape of an Italian cohort of adult onset ALS, providing the frequency of the four major ALS genes (C9orf72, SOD1, FUS, and TARDBP) and of others rare ones associated with ALS.

Adding up all the molecular analyses, we identified 55 patients with a causative o possibly causative variant in the four major ALS genes, 22 with a potentially pathogenic variant in rare ALS/FTD genes (novel likely pathogenic variants or previously reported variants in ALS patients), and 9 with variants defined as of uncertain significance (novel predicted to be likely pathogenic in genes linked to other neurodegenerative diseases or in ALS risk factor genes or heterozygous variants in ALS recessive genes), (Fig. 2a,b). Of these patients, five carried more than one variant, including one with a RE in the C9orf72 gene. In summary, $27.0 \%$ of the overall cohort carried an ALS-related variant, affecting $54.8 \%$ of fALS, and $17.5 \%$ of sALS patients (Fig. 2a,b).

Despite differences in the NGS panel and pipeline used to classify variants, these results are consistent with those reported in other high-throughput sequencing studies performed in the ALS Italian population [10, 33].

A significant proportion (45\%) of fALS patients remained without a genetic diagnosis, suggesting that other ALS genes may be involved and remain to be uncovered, possibly including, as for Alzheimer's Diseases, rare family private pathogenic variants in risk factor genes [39-41].

For the correct application of genetic testing, counseling for each patient and the interpretation of results, family history, pedigree analysis, and risk assessment remain crucial [42]. The family history for ALS in our cohort, affecting 
9.1\% of the cohort, was comparable to that of previous studies $[43,44]$. However, when the family history for other neurodegenerative diseases was also considered, as recommended [38], the rate increased to $25.5 \%$.

The two types of family history followed two distinct trends associated with AAO (Fig. 1b), where the family history for ALS (fALS-ALS) was associated with a lower AAO, and those with fALS-ND (family history for other neurodegenerative diseases, i.e., dementia and parkinsonism) did not differ from sALS (Fig. S1), suggesting the involvement of distinct pathogenic mechanisms and/or genetic backgrounds in these two overlapping clinical syndromes [45-47].

As for the genetic landscape, the overall mutation frequency of the four major ALS genes was $41.7 \%$ in fALS and $8.1 \%$ in sALS, similar to those observed in a recent metaanalysis [48]. These explained $76.6 \%$ of fALS-ALS cases, but only $22.2 \%$ of fALS-ND patients. The RE in C9orf72 gene, the most frequent pathogenic mutation in our sample (Table 2) and in general in the ALS/FTD continuum in Europe [47], was associated with every phenotype of the ALS/FTD continuum and was evenly distributed in the two subgroups of fALS (Table 2).

In contrast, SOD1 mutations were identified only in fALS-ALS (26.7\% of cases), and, similarly, mutations in FUS and TARDBP were preferentially linked to fALS-ALS (Table 2).

Concerning other genes (Table S1) the most frequently involved were those belonging to the ALS/FTD continuum, rarely causative of fALS forms [11]. Their variations were detected in 32 patients (15.5\% of the analyzed samples). Among these, SQSTMI proved to be the most frequently mutated gene, confirming previous finding in the Italian population [11] ( $n=7,3.4 \%$, Table 3), followed by $C H M P 2 B$ $(n=4,1.9 \%$, Table 3). SQSTM1 showed a behavior similar to that of RE C9orf72, sharing with the major ALS genes a young AAO of the affected patients, but with a rare family history for other neurodegenerative diseases (Tables 3, S6).

Other variants were found in $C C N F, C H C H D 10$, DCTN1, FIG4, OPTN, SETX, TBK1, and UBQLN1, and each represented less than $1 \%$ of our cohort, as previously reported $[10,11]$. Together, rare ALS/FTD genes accounted for $12 \%$ of our population.

Besides, eight other patients were carriers of a likely pathogenic variant in genes not related to the ALS/FTD continuum (APP, CSF1R, NOTCH3, ITM2B), but linked to other types of neurodegenerative dementia. According to the model that considers the neurodegenerative disease as a continuous variation in clinical/pathological features [49], these variants may be risk factors for ALS.

Recent studies highlighted the role of genetic risk factors in sALS patients, where heritability would represent about $21.0 \%$ [7]. Likewise, we found rare variants in the
NEK1 gene, all in sALS, in 5.8\% of the tested samples (3 patients out of 51) (Table 4).

Given the rarity of several mutations, we did not find any clinical features that allow us to define a specific screening pathway, as already highlighted in previous reports [42]. However, our data support the presence of at least two different pathogenetic components underlying the ALS phenotype based on the type of family history and suggest the implementation of a differentiated screening protocol.

As pointed out before, all ALS patients should be tested for RE in the C9Orf72 gene, given the extreme variability in familial occurrence and AAO, the relatively low cost of the test and the forthcoming of C9orf72-targeted therapeutic trials [47]. Furthermore, fALS-ALS patients should be screened for $S O D 1, F U S$, and TARDBP, which in our cohort explain $50 \%$ of fALS-ALS cases, reaching $76.6 \%$ with C9orf72 testing (Fig. 2).

In contrast, patients with a family history for other neurodegenerative diseases were more likely to carry a mutation in other ALS/FTD genes ( $20 \%$ vs. $4.1 \%$ in fALS-ALS corresponding to only one patient, \#ALS82, also carrying a SOD1 mutation) as an inheritable trait.

In our cohort, a pathogenic/probably pathogenic mutation in the four main ALS genes or the rare ALS/FTD genes was present in $8.1 \%$ and $10 \%$ of sALS, respectively (Fig. 2). fALS may resemble a sporadic disease due to incomplete penetration or incomplete family history. In the case of negative family history, some "red flags" such as early AAO ( $<50$ years), atypical rapid or slow progression of the disease, and the presence of dementia may suggest a familial form [50]. No other clinical features (ALS variant, dementia, etc.) have proven useful in suggesting the possible genetic origin.

Finally, since the diagnostic algorithms should be optimized according to ethnic origin, we confirm that in the Italian population SQSTMI should be analyzed together with the four main genes [11].

In conclusion, despite decades of intensive research, ALS etiology remains unexplained, and the number of genes associated with disease risk and pathogenesis continues to grow. An NGS approach or exome/genome studies are not yet exhaustive and are suggested for research, hoping that they may help find a tailor-made treatment option for most ALS patients in the future. We suggest a protocol that could be useful in a clinical setting.

Supplementary Information The online version contains supplementary material available at https://doi.org/10.1007/s00415-021-10521-w.

Acknowledgements The authors acknowledge all patients and their caregivers for supporting the research in ALS. They also thank the Italian Ministry of Research RFO, the "Fondazione del Monte", the "Fondazione Gino Galletti", and the "Fondazione Il Bene Onlus". Finally, 
we are greatful to the BoReALS group, for the clinical care of patients and for support in collecting data: Ilaria Bartolomei, Franca Cinelli, Arianna Cherici, Vitantonio Di Stasi, Rosaria Plasmati, Francesca Pastorelli, Cecilia Celidea Quarta, David Milletti, Raffaella Nasca, Francesca Rizzi, Luca Valeriani, Francesca Anzolin, Elisabetta Fantoni, Patrizia Avoni, Vincenzo Donadio, Fiorito Alessia, Sabina Capellari, Silvia de Pasqua, Giovanni Rizzo, Veria Vacchiano, Enrico Fileccia, Luca Albini-Riccioli, Piero Parchi, Maria Pia Foschini, Annalisa Pession, Stella Battaglia, Sofia Asioli, Luca Vignatelli, Michelangelo Stanzani-Maserati, Anna Bartoletti-Stella, Carolina Colombo, Serena Maselli, Maria Pia Giannoccaro, Fabrizio Salvi, Rocco Liguori.

Author contribution 1. Research project: A. Conception, B. Organization, C. Execution; 2. Statistical analysis: A. Design, B. Execution, C. Review and Critique; 3. Manuscript: A. Writing of the first draft, B. Review and Critique; Anna Bartoletti-Stella and Veria Vacchiano: 1A, 1B, 1C, 2A, 2B, and 3A. Rocco Liguori and Sabina Capellari: 1A, 1B, 1C, 2C, and 3B. Silvia De Pasqua, Giacomo Mengozzi, Dario De Biase, Ilaria Bartolomei, Patrizia Avoni, Giovanni Rizzo, Piero Parchi, Vincenzo Donadio, Adriano Chiò, Annalisa Pession, Federico Oppi, Fabrizio Salvi: 1C, 2C, 3B.

Funding Open access funding provided by Alma Mater Studiorum Università di Bologna within the CRUI-CARE Agreement.

Data availability The genetic data sets can be requested to the corresponding author. All clinical data are reported in the manuscript and in the supplementary data files.

\section{Declaration}

Conflicts of interest The authors declare no conflict of interest.

Financial disclosure R. Liguori reports personal fees from Biogen, Sanofi-Genzyme, Argon Healthcare s.r.1., Amicus Therapeutics s.r.l., and Alfasigma for Advisory Board consultancy and Lecture fees from Dynamicom Education, SIMG Service, Adnkronos salute unipersonale s.r.l., and DOC Congress s.r.l. outside the submitted work. The other authors do not have any disclosure to declare.

Ethics approval The study was conducted according to the revised Declaration of Helsinki and Good Clinical Practice guidelines and approved by the "Area Vasta Emilia Centro" ethics committee. Informed consent was given by study participants.

Open Access This article is licensed under a Creative Commons Attribution 4.0 International License, which permits use, sharing, adaptation, distribution and reproduction in any medium or format, as long as you give appropriate credit to the original author(s) and the source, provide a link to the Creative Commons licence, and indicate if changes were made. The images or other third party material in this article are included in the article's Creative Commons licence, unless indicated otherwise in a credit line to the material. If material is not included in the article's Creative Commons licence and your intended use is not permitted by statutory regulation or exceeds the permitted use, you will need to obtain permission directly from the copyright holder. To view a copy of this licence, visit http://creativecommons.org/licenses/by/4.0/.

\section{References}

1. Chiò A, Logroscino G, Traynor BJ et al (2013) Global epidemiology of amyotrophic lateral sclerosis: a systematic review of the published literature. Neuroepidemiology 41(2):118-130. https:// doi.org/10.1159/000351153

2. Strong MJ, Abrahams S, Goldstein LH et al (2017) Amyotrophic lateral sclerosis-frontotemporal spectrum disorder (ALS-FTSD): revised diagnostic criteria. Amyotroph Lateral Scler Frontotemporal Degener 18(3-4):153-174

3. Lattante S, Ciura S, Rouleau GA, Kabashi E (2015) Defining the genetic connection linking amyotrophic lateral sclerosis (ALS) with frontotemporal dementia (FTD). Trends Genet 31:263-273

4. Taylor JP, Brown RH Jr, Cleveland DW (2016) Decoding ALS: from genes to mechanism. Nature 539(7628):197-206

5. Shahheydari H, Ragagnin A, Walker AK et al (2017) Protein quality control and the amyotrophic lateral sclerosis/frontotemporal dementia continuum. Front Mol Neurosci 10:119

6. Brown C (2017) Non-familial ALS: a tangled web. Nature 550(7676):S109-S111. https://doi.org/10.1038/550S109a

7. Keller MF, Ferrucci L, Singleton AB et al (2014) Genome-wide analysis of the heritability of amyotrophic lateral sclerosis. JAMA Neurol 71(9):1123-1134

8. Al-Chalabi A, Fang F, Hanby MF et al (2010) An estimate of amyotrophic lateral sclerosis heritability using twin data. J Neurol Neurosurg Psychiatry 81(12):1324-1326

9. Corcia P, Couratier P, Blasco H et al (2017) Genetics of amyotrophic lateral sclerosis. Rev Neurol (Paris) 173(5):254-262

10. Lamp M, Origone P, Geroldi A et al (2018) Twenty years of molecular analyses in amyotrophic lateral sclerosis: genetic landscape of Italian patients. Neurobiol Aging 66:179.e5-179.e16

11. Pensato V, Magri S, Bella ED et al (2020) Sorting rare ALS genetic variants by targeted re-sequencing panel in Italian patients: OPTN, VCP, and SQSTM1 variants account for $3 \%$ of rare genetic forms. J Clin Med 9(2):412

12. Yamashita S, Ando Y (2015) Genotype-phenotype relationship in hereditary amyotrophic lateral sclerosis. Transl Neurodegener 4:13

13. Giannoccaro MP, Bartoletti-Stella A, Piras S et al (2017) Multiple variants in families with amyotrophic lateral sclerosis and frontotemporal dementia related to $\mathrm{C} 9$ orf 72 repeat expansion: further observations on their oligogenic nature. J Neurol 264(7):1426-1433

14. Marangi G, Traynor BJ (2015) Genetic causes of amyotrophic lateral sclerosis: new genetic analysis methodologies entailing new opportunities and challenges. Brain Res 1607:75-93

15. Brooks BR, Miller RG, Swash M, Munsat TL, World Federation of Neurology Research Group on Motor Neuron Diseases (2000) El Escorial revisited: revised criteria for the diagnosis of amyotrophic lateral sclerosis. Amyotroph Lateral Scler Other Motor Neuron Disord 1(5):293-299

16. Swinnen B, Robberecht W (2014) The phenotypic variability of amyotrophic lateral sclerosis. Nat Rev Neurol 10(11):661-670. https://doi.org/10.1038/nrneurol.2014.184

17. Chiò A, Moglia C, Canosa A et al (2020) ALS phenotype is influenced by age, sex, and genetics: a population-based study. Neurology 94(8):e802-e810. https://doi.org/10.1212/WNL.0000000000 008869

18. Dubois B, Slachevsky A, Litvan I, Pillon B (2000) The FAB: a frontal assessment battery at bedside. Neurology 55(11):1621-1626

19. Gallassi R, Lenzi P, Stracciari A et al (1986) Neuropsychological assessment of mental deterioration: purpose of a brief battery and a probabilistic definition of "normality" and "non-normality." Acta Psychiatr Scand 74:62-67 
20. Boylan K (2015) Familial amyotrophic lateral sclerosis. NeurolClin 33(4):807-830

21. Visani M, de Biase D, Bartolomei I et al (2011) A novel T137A SOD1 mutation in an Italian family with two subjects affected by amyotrophic lateral sclerosis. Amyotroph Lateral Scler 12(5):385-388

22. Bartoletti-Stella A, Baiardi S, Stanzani-Maserati M et al (2018) Identification of rare genetic variants in Italian patients with dementia by targeted gene sequencing. Neurobiol Aging 66:180.e23-180. e31

23. Karczewski KJ, Francioli LC, Tiao G et al (2020) The mutational constraint spectrum quantified from variation in 141,456 humans. Nature 581(7809):434-443

24. Richards S, Aziz N, Bale S et al (2015) Standards and guidelines for the interpretation of sequence variants: a joint consensus recommendation of the American College of Medical Genetics and Genomics and the Association for Molecular Pathology. Genet Med 17(5):405-424

25. Turner MR, Barnwell J, Al-Chalabi A, Eisen A (2012) Young-onset amyotrophic lateral sclerosis: historical and other observations. Brain 135(Pt 9):2883-2891

26. Espay AJ, Litvan I (2011) Extrapyramidal syndrome and frontotemporal dementia: the clinical overlap. J Mol Neurosci 45(3):343-349

27. Iacoangeli A, Al Khleifat A, Jones AR et al (2019) C9orf72 intermediate expansions of 24-30 repeats are associated with ALS. Acta Neuropathol Commun 7(1):115

28. Gellera C, Castellotti B, Riggio MC et al (2001) Superoxide dismutase gene mutations in Italian patients with familial and sporadic amyotrophic lateral sclerosis: identification of three novel missense mutations. Neuromuscul Disord 11(4):404-410

29. Gamez J, Corbera-Bellalta M, Nogales G et al (2006) Mutational analysis of the $\mathrm{Cu} / \mathrm{Zn}$ superoxide dismutase gene in a Catalan ALS population: should all sporadic ALS cases also be screened for SOD1? J Neurol Sci. 247(1):21-28

30. Giannini F, Battistini S, Mancuso M et al (2010) D90A-SOD1 mutation in ALS: the first report of heterozygous Italian patients and unusual findings. Amyotroph Lateral Scler 11(1-2):216-219

31. Hosler BA, Nicholson GA, Sapp PC et al (1996) Three novel mutations and two variants in the gene for $\mathrm{Cu} / \mathrm{Zn}$ superoxide dismutase in familial amyotrophic lateral sclerosis. Neuromuscul Disord 6(5):361-366

32. Dong SQ, Liu XN, Yang WB, Zhou YN, Wang JC, Chen XJ (2020) An exon 5 mutation (c.425G $>$ C, p.Gly141Ala) in the SOD1 gene in a Chinese family associated with incomplete penetrance [published online ahead of print, 2020 Mar 15]. Amyotroph Lateral Scler Frontotemporal Degener1-4

33. Lattante S, Conte A, Zollino M et al (2012) Contribution of major amyotrophic lateral sclerosis genes to the etiology of sporadic disease. Neurology 79(1):66-72

34. Münch C, Rolfs A, Meyer T (2008) Heterozygous S44L missense change of the spastin gene in amyotrophic lateral sclerosis. Amyotroph Lateral Scler 9(4):251-253

35. Ferrari R, Kapogiannis D, Huey ED, Momeni P (2011) FTD and ALS: a tale of two diseases. Curr Alzheimer Res 8(3):273-294
36. Cappella M, Ciotti C, Cohen-Tannoudji M, Biferi MG (2019) Gene therapy for ALS-A perspective. Int J Mol Sci 20(18):4388

37. Gibson SB, Downie JM, Tsetsou S et al (2017) The evolving genetic risk for sporadic ALS. Neurology 89(3):226-233

38. Vajda A, McLaughlin RL, Heverin M et al (2017) Genetic testing in ALS: a survey of current practices. Neurology 88(10):991-999

39. De Roeck A, Van Broeckhoven C, Sleegers K (2019) The role of ABCA7 in Alzheimer's disease: evidence from genomics, transcriptomics and methylomics. Acta Neuropathol 138(2):201-220

40. Nguyen HP, Van Broeckhoven C, van der Zee J (2018) ALS genes in the genomic era and their implications for FTD. Trends Genet 34(6):404-423

41. Renton AE, Chiò A, Traynor BJ (2014) State of play in amyotrophic lateral sclerosis genetics. Nat Neurosci 17(1):17-23

42. Perrone B, Conforti FL (2020) Common mutations of interest in the diagnosis of amyotrophic lateral sclerosis: how common are common mutations in ALS genes? [published online ahead of print, 2020 Jun 16]. Expert Rev Mol Diagn 1-12

43. Logroscino G, Traynor BJ, Hardiman O et al (2010) Incidence of amyotrophic lateral sclerosis in Europe. J Neurol Neurosurg Psychiatry 81(4):385-390

44. Masrori P, Van Damme P (2020) Amyotrophic lateral sclerosis: a clinical review [published online ahead of print, 2020 Jun 11]. Eur J Neurol. https://doi.org/10.1111/ene.14393

45. Tan RH, Ke YD, Ittner LM, Halliday GM (2017) ALS/FTLD: experimental models and reality. Acta Neuropathol 133(2):177-196

46. Karch CM, Wen N, Fan CC et al (2018) Selective genetic overlap between amyotrophic lateral sclerosis and diseases of the frontotemporal dementia spectrum. JAMA Neurol 75(7):860-875

47. Roggenbuck J, Quick A, Kolb SJ (2017) Genetic testing and genetic counseling for amyotrophic lateral sclerosis: an update for clinicians. Genet Med 19(3):267-274

48. Zou ZY, Zhou ZR, Che CH, Liu CY, He RL, Huang HP (2017) Genetic epidemiology of amyotrophic lateral sclerosis: a systematic review and meta-analysis. J Neurol Neurosurg Psychiatry 88(7):540-549

49. Armstrong RA (2012) On the "classification" of neurodegenerative disorders: discrete entities, overlap or continuum? Folia Neuropathol 50(3):201-208

50. Ajroud-Driss S, Siddique T (2015) Sporadic and hereditary amyotrophic lateral sclerosis (ALS). Biochim Biophys Acta 1852(4):679-684

51. Yilmaz R, Müller K, Brenner D et al (2020) SQSTM1/p62 variants in 486 patients with familial ALS from Germany and Sweden. Neurobiol Aging 87:139.e9-139.e15

52. Giannoccaro MP, Bartoletti-Stella A, Piras S et al (2018) The first historically reported Italian family with FTD/ALS teaches a lesson on C9orf72 RE: clinical heterogeneity and oligogenic inheritance. J Alzheimers Dis 62(2):687-697. https://doi.org/10.3233/ JAD-170913

53. D'Amore A, Tessa A, Casali C et al (2018) Next generation molecular diagnosis of hereditary spastic paraplegias: an Italian cross-sectional study. Front Neurol 9:981 